\title{
Hierarchical-clustering-based Cokriging to advance the spatial prediction accuracy of soil heavy metals distributions
}

\author{
LING ZENG ${ }^{1}$, JASON HERRIN ${ }^{2}$
}

123

${ }^{1}$ Geomathematics Key Laboratory of Sichuan Province, Chengdu Technological University, Chengdu, 610059, China and zengling18@cdut.edu.cn

2 Facility for Analysis Characterization Testing Simulation, Nanyang Technological University, Singapore 639798, Singapore and JSHerrin@ntu.edu.sg

Improving the understanding and characterization of spatial soil heavy metal distribution is becoming an important component of risk assessment and environmental policy. Geostatistical analysis methods are the most commonly used means to interpolate soil heavy metals spatial distributions. Many researches take the correlation with other environment variables, such as soil properties, into the estimation of soil heavy metals distributions. However, choices of environment variables were often determined by empirical knowledge. Actually each of the eight heavy metals may have different correlation with other environmental chemicals in different geographical backgrounds. Hierarchical clustering method will assist to quantify the level of correlation of environmental variables with each soil heavy metal, and thus determine the explanatory variables to estimate each soil heavy metals by cokriging method. In this work, 196 soil samples collected from Youyang (Chongqing, China) were used as the empirical dataset. By comparison with ordinary cokriging and geographically weighted regression, we analyse the validation of hierarchical-clustering-based cokriging. 